\title{
A prospective study of intake of trans-fatty acids from ruminant fat, partially hydrogenated vegetable oils, and marine oils and mortality from CVD
}

\author{
Ida Laake ${ }^{1 *}$, Jan I. Pedersen ${ }^{2}$, Randi Selmer ${ }^{3}$, Bente Kirkhus $^{4}$, Anja S. Lindman ${ }^{5}$, Aage Tverdal ${ }^{3}$ \\ and Marit B. Veierød ${ }^{1,2}$ \\ ${ }^{1}$ Department of Biostatistics, Institute of Basic Medical Sciences, University of Oslo, Boks 1122 Blindern, O317 Oslo, Norway \\ ${ }^{2}$ Department of Nutrition, Institute of Basic Medical Sciences, University of Oslo, Oslo, Norway \\ ${ }^{3}$ Division of Epidemiology, The Norwegian Institute of Public Health, Oslo, Norway \\ ${ }^{4}$ Nofima AS, Norwegian Institute of Food, Fisheries and Aquaculture Research, As, Norway \\ ${ }^{5}$ Norwegian Knowledge Centre for the Health Services, Oslo, Norway \\ (Submitted 10 March 2011 - Final revision received 27 September 2011 - Accepted 30 September 2011 - First published online 8 November 2011)
}

\section{Abstract}

Trans-fatty acids (TFA) have adverse effects on blood lipids, but whether TFA from different sources are associated with risk of CVD remains unresolved. The objective of the present study was to evaluate the association between TFA intake from partially hydrogenated vegetable oils (PHVO), partially hydrogenated fish oils (PHFO) and ruminant fat (rTFA) and risks of death of CVD, CHD, cerebrovascular diseases and sudden death in the Norwegian Counties Study, a population-based cohort study. Between 1974 and 1988 , participants were examined for up to three times. Fat intake was assessed with a semi-quantitative FFQ. A total of 71464 men and women were followed up through 2007. Hazard ratios (HR) and 95\% CI were estimated with Cox regression. Energy from TFA was compared to energy from all other sources, carbohydrates or unsaturated cis-fatty acids with different multivariable models. During follow-up, 3870 subjects died of CVD, 2383 of CHD, 732 of cerebrovascular diseases and 243 of sudden death. Significant risks, comparing highest to lowest intake category, were found for: TFA from PHVO and CHD (HR 1.23 (95\% CI 1.00, 1.50)) and cerebrovascular diseases (HR 0.65 (95\% CI 0.45, 0.94)); TFA from PHFO and CVD (HR 1.14 (95\% CI 1.03, 1.26)) and cerebrovascular diseases (HR 1.32 (95\% CI 1.04, 1.69)); and rTFA intake and CVD (HR 1.30 (95\% CI 1.05, 1.61)), CHD (HR 1.50 (95\% CI 1.11, 2.03)) and sudden death (HR 2.73 (95\% CI 1.19, 6.25)) in women. These associations with rTFA intake were not significant in men $\left(P_{\text {interaction }} \geq 0.01\right)$. The present study supports that TFA intake, irrespective of source, increases CVD risk. Whether TFA from PHVO decreases risk of cerebrovascular diseases warrants further investigation.

\section{Key words: Trans-fatty acids: CVD: CHD: Cohort studies}

There is general agreement that trans-fatty acids (TFA) in the diet have contributed to the CHD epidemic during the last three quarters of a century ${ }^{(1,2)}$. Dietary TFA are derived from three main sources: partially hydrogenated vegetable oils (PHVO) and partially hydrogenated fish oils (PHFO), collectively named industrially formed TFA, and from ruminant fat, formed in the rumen by bio-hydrogenation of unsaturated fatty acids.

TFA increase LDL-cholesterol and reduce HDL-cholesterol. This has been documented for $\mathrm{PHVO}^{(3)}$ and for $\mathrm{PHFO}^{(4)}$, as well as for ruminant trans-fatty acid (rTFA) ${ }^{(5,6)}$. Some studies have also found that TFA increase $\operatorname{Lp}(\mathrm{a})^{(4,7)}$. These metabolic effects may, to a large extent, explain that TFA are related to increased risk of CHD death ${ }^{(8)}$. TFA may also be related to risk beyond the effect on plasma cholesterol ${ }^{(1,9)}$.
PHFO were used in considerable amounts in the United Kingdom, the Netherlands, Germany, the Soviet Union and Norway $^{(10)}$, all countries with a high CHD mortality. In 1986 , the daily per capita consumption of PHFO was $11 \mathrm{~g}$ in Great Britain, $24 \mathrm{~g}$ in the Netherlands and $10 \mathrm{~g}$ in West Germany ${ }^{(4)}$. In 1960, the consumption of margarine in Norway was $66 \mathrm{~g} / \mathrm{d}$. Of the fat used for production, $73 \%$ was derived from partially hydrogenated oil, of which $98 \%$ was from $\mathrm{PHFO}$ and $2 \%$ from $\mathrm{PHVO}^{(11)}$. Margarine consumption was gradually reduced to $36 \mathrm{~g} / \mathrm{d}$ in 1994 . At that time, PHFO and PHVO provided approximately 10 and $5 \%$ of total fat intake, respectively $^{(4)}$. A study on the association between TFA from PHFO and risk of death from CVD will contribute to establish causes of the CHD epidemic. The very high former intake

Abbreviations: E\%, energy percentage; HR, hazard ratio; PHFO, partially hydrogenated fish oils; PHVO, partially hydrogenated vegetable oils; rTFA, ruminant trans-fatty acids; TFA, trans-fatty acids.

*Corresponding author: I. Laake, fax +47 228513 13, email ida.laake@medisin.uio.no 
of TFA from PHFO makes the Norwegian population particularly suitable for studies of the risk of CVD associated with these fatty acids.

Practically all epidemiological studies on the association between TFA intake and CVD risk are based on TFA from $\mathrm{PHVO}^{(1)}$. No study has so far been undertaken on the association between intake of TFA from PHFO and CVD risk. Moreover, only a few studies have examined the association between rTFA intake and CVD risk ${ }^{(12-16)}$, and the results are conflicting. Inverse associations, significant ${ }^{(14)}$ and nonsignificant $^{(12)}$, have been reported. Others have found no association $^{(13,16)}$ or a weak, non-significant, positive association $^{(15)}$. Significant positive associations have not yet been found, even though rTFA seem to have adverse effects on blood lipids ${ }^{(5,6)}$. Whether rTFA intake is associated with risk of CVD is thus still unresolved.

The Norwegian Counties Study is a large cohort study with detailed information on sources and amounts of dietary fat intake. Through information from the margarine industry on the composition of the different margarines, we have been able to quantify the intake of TFA from different sources. The aim of the present study is to examine the associations between intake of TFA from PHVO, PHFO and ruminant fat and mortality from CVD and CHD.

\section{Subjects and methods}

\section{The Norwegian Counties Study}

The Norwegian Counties Study was initiated by the National Health Screening Service to identify risk factors and screen for CVD. Between 1974 and 1988, three screenings were carried out in the counties Finnmark, Oppland and Sogn og Fjordane. All residents aged 35-49years and a random sample of persons aged 20-34 years, in total 65624 persons, were invited to screening $\mathrm{I}^{(17)}$. Everyone invited to screening I still residing in the county was re-invited to screening II. At screening III, the majority of persons invited to screening II were re-invited, but in the oldest age group ( $\geq 55$ years) only a random sample was re-invited. Screenings II and III also included random samples of younger persons not invited to previous screenings, $17-39$ years and 20-39 years, respectively. Time between subsequent screenings was 5 years in Oppland and Sogn og Fjordane, and 3 and 13 years, respectively, in Finnmark. The attendance was 88, 88 and $84 \%$ at screenings I, II and III, respectively. A total of 92234 persons attended at least one screening.

The procedures were the same at each screening. A questionnaire covering smoking habits, recreational and occupational physical activity and history and symptoms of CVD was included with the invitation. The completed questionnaire was brought to the examination where it was checked for inconsistencies. A team of specially trained nurses carried out the examinations. The participants' blood pressure was measured (twice at screenings I and II, three times at screening III). Height and weight were measured to the nearest centimetre and half kilogram. Also, a non-fasting blood sample was collected and analysed for TAG and total cholesterol. A four-page semi-quantitative FFQ to be filled out at home and returned by mail in a pre-paid envelope was handed out. At screening I, the FFQ was not included in all municipalities.

Information on the participants' level of attained education was obtained from Statistics Norway: using the unique elevendigit identification number that all Norwegian citizens are assigned, the participants were linked to records of the Censuses of 1970, 1980, 1990 and 2001.

The present study was approved by the Norwegian Data Inspectorate and recommended by the Regional Committee for Medical Research Ethics.

\section{Assessment of dietary intake}

A detailed description of the FFQ and its reliability, validity and the method for calculating nutrient intakes has been published previously ${ }^{(18)}$. The FFQ covered intake of eighty food items, with special emphasis on sources of fat in the Norwegian diet. Food items contributing in the calculations of nutrient intake included bread, fats on bread, spreads, cheese, milk, cakes, eggs, meat and fish dishes, potatoes and cod liver oil. For most food items, we used standard portion sizes. The nutrient content in the food items was obtained from the Norwegian food composition tables, from the time that the data were collected. Intake of TFA was calculated using the information provided by the margarine industry on the composition of retail margarines and fats used by the baking industry at the time of the screenings. All margarines and fats were produced in Norway. PHFO and PHVO used for production of margarines and fats were provided by a single producer (A/S Denofa og Lilleborg Fabrikker, Fredrikstad, Norway) and derived from fish oil and soyabean oil, respectively. The melting point of PHVO was $40 / 42^{\circ} \mathrm{C}$. Different fish oils, depending on availability, were mixed to give an iodine number of 140 . For margarine production, two different types of PHFO with melting points of $30 / 32^{\circ} \mathrm{C}$ and $38 / 40^{\circ} \mathrm{C}$ were used. Standard hydrogenation conditions were used: temperature, $155^{\circ} \mathrm{C}$ (PHVO) or $155-180^{\circ} \mathrm{C}$ (PHFO); catalyst, nickel; and pressure, 0.5-1.0 bar above atmospheric pressure. The TFA content of the hydrogenated fractions was determined by capillary liquid GC combined with $\mathrm{TLC}^{(4)}$, and the TFA content in products was calculated based on these data and the recipes. When applicable, figures on TFA levels of foods collected in the TRANSFAIR study were used $^{(19)}$

We calculated intake of TFA from PHFO and PHVO based on the amount and type of margarine consumed. We also calculated rTFA intake based on consumption of dairy products and ruminant meat products.

We did not calculate daily nutrient intakes if a subject left more than ten questions unanswered. The FFQ was handed out to $59 \%$ of the participants at screening I and to all at screenings II and III. Of these, nutrient intakes could be estimated for 93, 82 and $84 \%$, respectively. We eliminated intakes if the ratio of energy intake to the $\mathrm{BMR}^{(20)}$ was in the screening-specific top and bottom $1 \%$. 


\section{Follow-up and end points}

Follow-up started at a subject's baseline screening, which we defined as the first screening in which our requirements regarding daily nutrient intakes were fulfilled, age $\geq 18$ years, the questions about smoking were answered, a blood sample was collected and measurements of blood pressure and BMI were obtained. Thus, start of follow-up for a subject may be later than the first attended screening. The subjects were followed up until death, emigration or 31 December 2007. Information on death and emigration was obtained through linkage with Statistics Norway using the unique identification number. We studied death by the following causes: all causes, CVD (ICD 8: 390-458; ICD 9: 390-459; ICD 10: I00-I99), CHD (ICD 8-9: 410-414; ICD 10: I20I25), cerebrovascular diseases (ICD 8-9: 430-438; ICD 10: I60-I69) and sudden death of unknown cause (ICD 8: 782·4, 795; ICD 9: 798·1, 798·2; ICD 10: R96).

\section{Study sample}

After eliminating very high and low intakes, we had information on fat intake from at least one screening for 79227 subjects (Fig. 1). The analyses were restricted to subjects assigned a baseline screening (see above). We excluded subjects who reported having or having had heart infarction, angina pectoris, arteriosclerosis of the legs, cerebral stroke, other heart diseases or diabetes before start of follow-up and subjects with symptoms of angina pectoris at baseline. We also excluded subjects without information on education. In total, 71464 subjects $(50 \cdot 3 \%$ men) were included in our analyses (Fig. 1). Of these, 34109 had one measurement of nutrient intake, 22144 had two measurements and 15211 three measurements.

\section{Statistical analyses}

All nutrient intakes are given as percentage of total energy intake (\% of $\mathrm{kJ} / \mathrm{d}=\mathrm{E} \%$ ). We categorised the intakes of TFA at each screening as follows: <0.15, 0.15-0.649, 0.65-1.149, $1.15-1.649$ and $\geq 1.65 \mathrm{E} \%$ for TFA from PHVO and $<0.85$, $0.85-1.349,1.35-1.849,1.85-2.349$ and $\geq 2.35 \mathrm{E} \%$ for TFA from PHFO. Intake of rTFA at each screening was categorised as $<0.4,0.4-0.549,0.55-0.699,0.7-0.849$ and $\geq 0.85 \mathrm{E} \%$. TFA intake cutoff points were chosen such that the category intervals were of equal length, while ensuring a sufficient number of cases in each category. We did not use percentiles, because of the changes in intake of TFA that occurred over time. At each screening, we classified the participants as current,

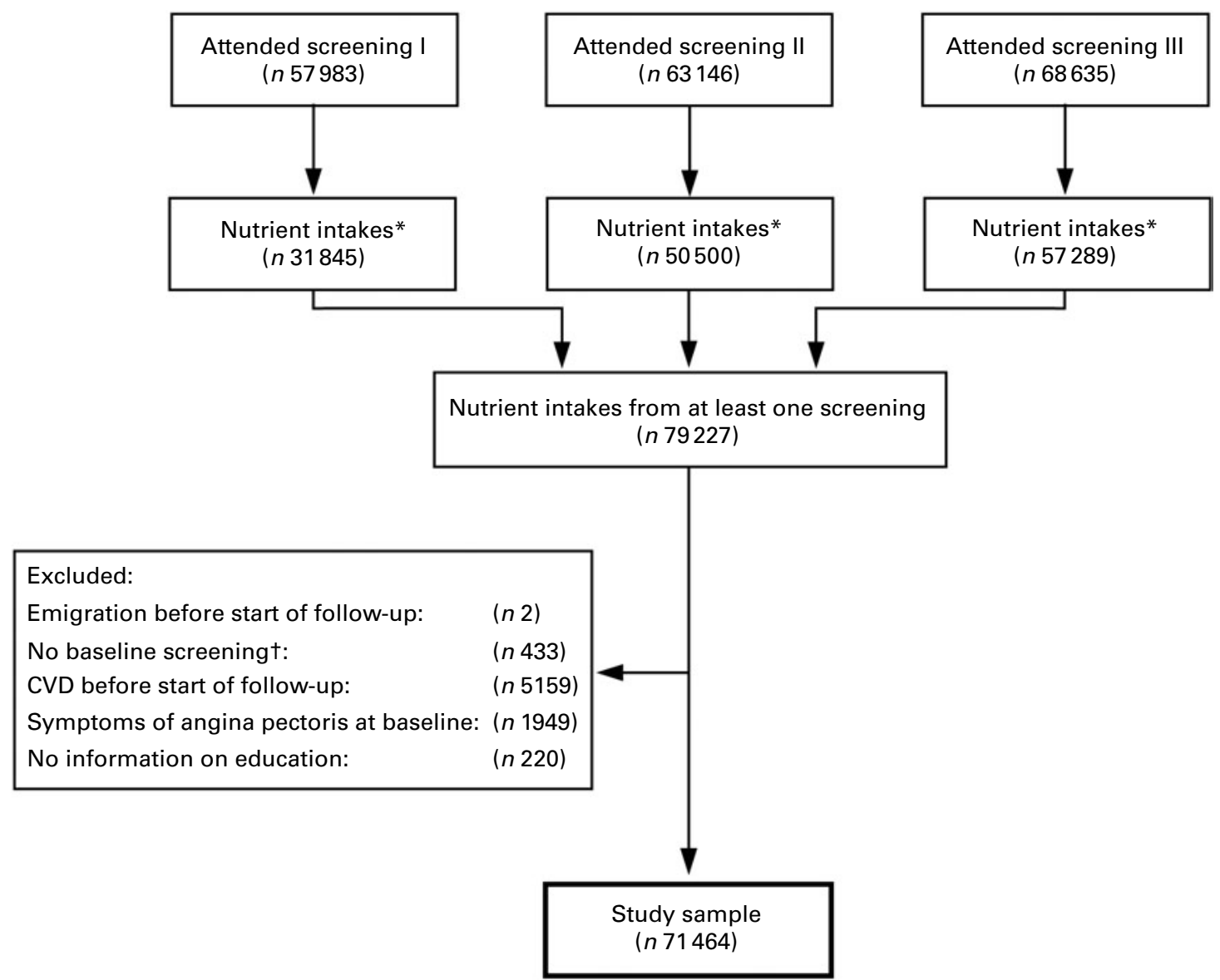

Fig. 1. Flow chart illustrating how the study sample was obtained. *Intakes were not calculated for participants with $>10$ unanswered questions on the FFQ. Intakes were eliminated if the ratio of energy intake to the basal metabolic rate was in the top or bottom $1 \%$. † Baseline screening is defined as the first attended screening where information on diet, smoking and BMI was obtained, blood pressure was measured, blood sample was collected, and age $\geq 18$ years. 
former and never smokers. Participants were classified as former instead of never smokers at a screening if they had been classified as former or daily smokers at a previous screening. We used the second measurement of systolic blood pressure from screenings I and II, and the mean of the second and third measurements from screening $\mathrm{III}^{(21)}$. Each participant was classified as having primary schooling ( $\leq 9$ years), secondary education (10-12 years) or education on university level ( $\geq 13$ years), using the most recent information available from the censuses.

To examine the relationship between intake of TFA and risk of death, we used Cox regression ${ }^{(22)}$ and estimated hazard ratios (HR) and 95\% CI. All models were adjusted for sex and for age by using attained age as the time variable and by controlling for year of birth (<1930, 1930-4, 1935-9, $1940-4$ and $\geq 1945$ ) in a stratified Cox model. Three multivariable models were used. Model 1 was adjusted for total energy intake, systolic blood pressure, BMI (all continuous), smoking and attained education. Model 2 was based on model 1, with additional adjustment for intakes of saturated fat, unsaturated cis-fatty acids, protein, all other TFA and cholesterol (all continuous). Model 3 was adjusted for the same variables as model 2, except that intake of carbohydrates was included in model 3 and intake of unsaturated cis-fatty acids was removed. As total energy intake is included in the multivariable models, these models compare the effect of energy from TFA to the effect of the same amount of energy from the nutrients not included in the model. Thus, energy from TFA is compared to energy from all other sources in model 1, energy from carbohydrates in model 2 and in model 3 TFA is compared to energy from unsaturated cisfatty acids.

All nutrient intakes, smoking, BMI and blood pressure were modelled as time-dependent variables. Values were updated at each attended screening where information on all covariates was complete. At each time point, we used values from the most recent preceding attended screening for smoking, blood pressure and BMI, whereas for the nutrient intakes, we used the mean of intakes from all preceding attended screenings since the start of follow-up ${ }^{(23)}$, that is, for a subject with start of follow-up at screening I and with complete information on all covariates from all three screenings, intake from screening I was used until screening II, the mean of intakes from screenings I and II was used between screenings II and III and the mean of all three intakes was used from screening III until the end of follow-up. If nutrient intake was measured only at baseline, this value was used throughout follow-up.

To test for linear trends, the exposure was modelled as a continuous variable. We also tested for interaction between TFA intake and sex. Furthermore, the association between TFA intake and CHD risk may differ by BMI category ${ }^{(24)}$; thus, we tested for interaction between TFA intake and BMI $\left(<25\right.$ or $\left.\geq 25 \mathrm{~kg} / \mathrm{m}^{2}\right)$. Because of the long follow-up, we also tested for interaction between TFA intake and follow-up time $(<10$ or $\geq 10$ years since last measurement of nutrient intake). Interaction tests were based on both model 2 and model 3, with TFA intake modelled continuously.
We used a likelihood ratio test to test for interaction and linear trend in models without interaction. In models with interaction, a Wald test was used to test for linear trend. If we found significant interaction between TFA intake and a variable, we stratified on the variable when performing interaction tests on TFA intake and the other variables. Tests of statistical significance were two-sided and $P<0.05$ was considered statistically significant. The analyses were performed using SAS version 9.2 (SAS Institute, Cary, NC, USA).

\section{Results}

Mean follow-up was 25.8 years. During follow-up, 11890 subjects died, 3870 of CVD, 2383 of CHD, 732 of cerebrovascular diseases and 243 deaths were classified as sudden of unknown cause.

\section{Industrial trans-fatty acids}

At baseline, mean intakes of TFA from PHVO and PHFO were 0.9 and $1.6 \mathrm{E} \%$, respectively. For TFA from PHVO, men were in the majority in the category with the highest intake (Table 1). Mean intake of rTFA decreased, and mean intake of polyunsaturated cis-fatty acids increased with increasing category of intake of TFA from PHVO. Serum cholesterol was not related to intake of TFA from PHVO. For TFA from PHFO, there was a majority of men among subjects with the lowest intake (Table 2). Furthermore, intake of polyunsaturated cis-fatty acids was not related to intake of TFA from PHFO. Mean dietary cholesterol and serum cholesterol levels increased with increasing intake of TFA from PHFO.

Intake of TFA from PHVO was significantly, positively related to risk of death from $\mathrm{CHD}$ with model 3, HR comparing highest to lowest category of intake was $1 \cdot 23$, 95\% CI 1.00, 1.50 (Table 3). Furthermore, intake was inversely related to risk of death from cerebrovascular diseases with both models 2 and 3 (model 3: HR comparing highest to lowest category of intake was $0.65,95 \%$ CI $0.45,0.94)$. There were no significant interactions between intake of TFA from PHVO and sex (model 3: $P_{\text {interaction }} \geq 0 \cdot 23$ ) or BMI (model 3: $P_{\text {interaction }} \geq 0 \cdot 15$ ). The association between intake of TFA from $\mathrm{PHVO}$ and risk of death differed significantly with follow-up for CVD, CHD and sudden death $\left(P_{\text {interaction }}=0.02\right.$, 0.02 and 0.05 , respectively, with model 3 ). With model 3 , HR per $0.5 \mathrm{E} \%$ increase in intake for follow-up time $<10$ and $\geq 10$ years after last measurement, respectively, were 1.04 (95\% CI $0.99,1.09)$ and $0.99(95 \%$ CI $0.95,1.02)$ for CVD; $1.09(95 \%$ CI $1.03,1.16)$ and 1.03 (95\% CI $0.98,1.08)$ for CHD; and 1.08 (95\% CI $0.92,1.28)$ and 0.93 (95\% CI $0.79,1.08)$ for sudden death. The interaction results were similar with model 2.

Intake of TFA from PHFO was significantly, positively associated with risk of death from CVD with model 1, HR comparing highest to lowest intake was $1 \cdot 14$, 95\% CI $1 \cdot 03$, 1.26 (Table 4). With models 2 and 3, we found similar effect estimates, but they were not significant. We found no significant association between intake and risk of death from CHD with either model. Intake was significantly, positively associated with risk of cerebrovascular diseases with model 1 
Table 1. Characteristics of study sample at baseline according to intake of trans-fatty acids (TFA) from partially hydrogenated vegetable oil (PHVO)

(Mean values and percentages)

\begin{tabular}{|c|c|c|c|c|c|}
\hline & \multicolumn{5}{|c|}{ Intake of TFA from PHVO at baseline (E\%) } \\
\hline & $<0.15$ & $0.15-0.649$ & $0.65-1.149$ & $1.15-1.649$ & $\geq 1.65$ \\
\hline Participants $(n)$ & 16649 & 18997 & 7343 & 15828 & 12647 \\
\hline Mean follow-up (years) & $25 \cdot 3$ & 25.9 & $25 \cdot 8$ & $25 \cdot 9$ & $26 \cdot 1$ \\
\hline Men (\%) & 51.5 & 43.0 & 34.5 & $49 \cdot 0$ & $70 \cdot 4$ \\
\hline Mean age at baseline (years) & $40 \cdot 6$ & 41.5 & $40 \cdot 4$ & $40 \cdot 7$ & $40 \cdot 6$ \\
\hline \multicolumn{6}{|l|}{ Baseline screening (\%) } \\
\hline Screening 1 & $39 \cdot 7$ & 44.4 & 39.5 & $36 \cdot 8$ & $38 \cdot 8$ \\
\hline Screening 2 & $22 \cdot 8$ & $26 \cdot 7$ & $30 \cdot 7$ & $31 \cdot 2$ & $34 \cdot 6$ \\
\hline Screening 3 & 37.5 & 28.8 & $29 \cdot 8$ & $32 \cdot 0$ & $26 \cdot 7$ \\
\hline Mean intake of TFA from PHFO (E\%) & 1.56 & $2 \cdot 15$ & 1.68 & 1.30 & $1 \cdot 12$ \\
\hline Mean ruminant TFA intake (E\%) & 0.68 & 0.65 & 0.53 & 0.49 & 0.46 \\
\hline Mean energy intake $(\mathrm{kJ} / \mathrm{d})$ & 7119 & 6728 & 6043 & 7106 & 8426 \\
\hline Mean total fat intake $(\mathrm{E} \%)$ & 35.5 & 38.6 & $37 \cdot 6$ & 37.5 & 41.6 \\
\hline Mean SFA intake (E\%) & $15 \cdot 9$ & $16 \cdot 6$ & $14 \cdot 7$ & $13 \cdot 9$ & $14 \cdot 3$ \\
\hline Mean monounsaturated cis-fatty acids intake ( $\mathrm{E} \%$ ) & $8 \cdot 8$ & $9 \cdot 4$ & $9 \cdot 4$ & $9 \cdot 2$ & $10 \cdot 2$ \\
\hline Mean polyunsaturated cis-fatty acids intake ( $\mathrm{E} \%)^{\prime}$ & 3.9 & 4.9 & $5 \cdot 7$ & $6 \cdot 6$ & 8.9 \\
\hline Mean carbohydrate intake (E\%) & 48.5 & $45 \cdot 7$ & $46 \cdot 2$ & $47 \cdot 0$ & $44 \cdot 3$ \\
\hline Mean protein intake $(\mathrm{E} \%)$ & $16 \cdot 0$ & $15 \cdot 7$ & $16 \cdot 2$ & $15 \cdot 5$ & $14 \cdot 1$ \\
\hline Mean cholesterol intake (mg/MJ) & 38.4 & $40 \cdot 8$ & 38.4 & 33.4 & $30 \cdot 4$ \\
\hline Mean systolic blood pressure (mmHg) & 132 & 131 & 131 & 131 & 133 \\
\hline Mean total serum cholesterol (mmol/l) & 6.04 & $6 \cdot 22$ & 6.05 & 5.96 & 6.00 \\
\hline Mean serum TAG $(\mathrm{mmol} / \mathrm{l})$ & 1.66 & 1.63 & 1.61 & 1.63 & 1.78 \\
\hline Daily smokers (\%) & $38 \cdot 8$ & $44 \cdot 3$ & $42 \cdot 6$ & $42 \cdot 1$ & $48 \cdot 8$ \\
\hline Mean BMI $\left(\mathrm{kg} / \mathrm{m}^{2}\right)$ & $24 \cdot 7$ & $24 \cdot 8$ & 24.5 & $24 \cdot 4$ & $24 \cdot 4$ \\
\hline Inactive (\%) & $22 \cdot 0$ & $25 \cdot 0$ & $26 \cdot 3$ & $25 \cdot 3$ & 24.9 \\
\hline Higher education (\%) & $15 \cdot 3$ & $13 \cdot 0$ & 12.5 & $15 \cdot 0$ & $12 \cdot 7$ \\
\hline
\end{tabular}

E\%, energy percentage; PHFO, partially hydrogenated fish oil.

Table 2. Characteristics of study sample at baseline according to intake of trans-fatty acids (TFA) from partially hydrogenated fish oil (PHFO)

(Mean values and percentages)

\begin{tabular}{|c|c|c|c|c|c|}
\hline & \multicolumn{5}{|c|}{ Intake of TFA from PHFO at baseline (E\%) } \\
\hline & $<0.85$ & $0.85-1.349$ & $1.35-1.849$ & $1 \cdot 85-2 \cdot 349$ & $\geq 2.35$ \\
\hline Participants $(n)$ & 16882 & 18879 & 13682 & 8366 & 13655 \\
\hline Mean follow-up (years) & $24 \cdot 8$ & 25.5 & $26 \cdot 0$ & 26.5 & $26 \cdot 8$ \\
\hline Men (\%) & $64 \cdot 6$ & $56 \cdot 4$ & $42 \cdot 0$ & 33.4 & $42 \cdot 9$ \\
\hline Mean age at baseline (years) & $40 \cdot 1$ & $40 \cdot 9$ & 41.3 & 41.3 & $41 \cdot 1$ \\
\hline \multicolumn{6}{|l|}{ Baseline screening (\%) } \\
\hline Screening 1 & 30.4 & $36 \cdot 0$ & 41.6 & $47 \cdot 1$ & $52 \cdot 1$ \\
\hline Screening 2 & $28 \cdot 3$ & 29.5 & $28 \cdot 2$ & $28 \cdot 7$ & $28 \cdot 0$ \\
\hline Screening 3 & $41 \cdot 2$ & 34.5 & $30 \cdot 2$ & 24.2 & $19 \cdot 9$ \\
\hline Mean intake of TFA from PHVO (E\%) & $1 \cdot 12$ & 1.09 & 0.95 & 0.78 & 0.46 \\
\hline Mean ruminant TFA intake (E\%) & 0.64 & 0.60 & 0.57 & 0.54 & 0.50 \\
\hline Mean energy intake $(\mathrm{kJ} / \mathrm{d})$ & 7687 & 7457 & 6718 & 6243 & 6961 \\
\hline Mean Total fat intake (E\%) & $37 \cdot 2$ & 37.7 & 37.7 & 38.0 & $40 \cdot 0$ \\
\hline Mean saturated fat intake ( $\mathrm{E} \%)$ & $15 \cdot 1$ & $15 \cdot 1$ & $15 \cdot 1$ & $15 \cdot 1$ & $15 \cdot 9$ \\
\hline Mean monounsaturated cis-fatty acids intake ( $\mathrm{E} \%$ ) & 9.5 & $9 \cdot 4$ & $9 \cdot 4$ & $9 \cdot 3$ & $9 \cdot 1$ \\
\hline Mean polyunsaturated cis-fatty acids intake (E\%) & 5.9 & $5 \cdot 8$ & $5 \cdot 6$ & 5.5 & $6 \cdot 1$ \\
\hline Mean carbohydrate intake (E\%) & $47 \cdot 2$ & $46 \cdot 8$ & $46 \cdot 5$ & $46 \cdot 2$ & $45 \cdot 0$ \\
\hline Mean protein intake $(\mathrm{E} \%)$ & $15 \cdot 6$ & $15 \cdot 6$ & $15 \cdot 7$ & $15 \cdot 8$ & $15 \cdot 0$ \\
\hline Mean cholesterol intake (mg/MJ) & 33.4 & $35 \cdot 1$ & $37 \cdot 2$ & $39 \cdot 0$ & $40 \cdot 2$ \\
\hline Mean systolic blood pressure $(\mathrm{mmHg})$ & 132 & 132 & 131 & 131 & 131 \\
\hline Mean total serum cholesterol $(\mathrm{mmol} / \mathrm{l})$ & 5.96 & 6.02 & 6.04 & $6 \cdot 11$ & 6.25 \\
\hline Mean serum TAG $(\mathrm{mmol} / \mathrm{l})$ & 1.75 & 1.70 & 1.60 & 1.55 & 1.64 \\
\hline Daily smokers (\%) & $47 \cdot 8$ & 43.3 & 38.9 & 38.5 & 44.5 \\
\hline Mean BMI $\left(\mathrm{kg} / \mathrm{m}^{2}\right)$ & 24.5 & 24.5 & 24.5 & $24 \cdot 6$ & $24 \cdot 7$ \\
\hline Inactive (\%) & $22 \cdot 4$ & 24.4 & $25 \cdot 8$ & $26 \cdot 5$ & 24.5 \\
\hline Higher education (\%) & $15 \cdot 2$ & $15 \cdot 4$ & $15 \cdot 4$ & 13.1 & $9 \cdot 1$ \\
\hline
\end{tabular}

E\%, energy percentage; PHVO, partially hydrogenated vegetable oil. 
Table 3. Hazard ratio (HR) and $95 \% \mathrm{Cl}$ of cause-specific death according to intake of trans-fatty acids (TFA) from partially hydrogenated vegetable oil (PHVO) (Number of deaths, hazard ratios and $95 \%$ confidence intervals)

\begin{tabular}{|c|c|c|c|c|c|c|c|c|c|c|c|}
\hline & \multicolumn{11}{|c|}{ Intake of TFA from PHVO (E\%) } \\
\hline & \multicolumn{2}{|c|}{$<0 \cdot 15$} & \multicolumn{2}{|c|}{$0.15-0.649$} & \multicolumn{2}{|c|}{$0.65-1 \cdot 149$} & \multicolumn{2}{|c|}{$1 \cdot 15-1 \cdot 649$} & \multicolumn{2}{|c|}{$\geq 1.65$} & \multirow[b]{2}{*}{$P$} \\
\hline & $\mathrm{HR}$ & $95 \% \mathrm{Cl}$ & $\mathrm{HR}$ & $95 \% \mathrm{Cl}$ & HR & $95 \% \mathrm{Cl}$ & $\mathrm{HR}$ & $95 \% \mathrm{Cl}$ & $\mathrm{HR}$ & $95 \% \mathrm{Cl}$ & \\
\hline Person years & \multicolumn{2}{|c|}{421947} & \multicolumn{2}{|c|}{492806} & \multicolumn{2}{|c|}{189709} & \multicolumn{2}{|c|}{409685} & \multicolumn{2}{|c|}{329562} & \\
\hline \multicolumn{12}{|l|}{ All causes } \\
\hline Deaths $(n)$ & \multicolumn{2}{|c|}{2552} & \multicolumn{2}{|r|}{3401} & \multicolumn{2}{|c|}{1198} & \multicolumn{2}{|c|}{2395} & \multicolumn{2}{|c|}{2344} & \\
\hline Age-adjusted* & 1 & Ref & $1 \cdot 10$ & $1.04,1 \cdot 16$ & 1.12 & $1.06,1 \cdot 19$ & 1.00 & $0.94,1.06$ & 1.08 & $1.02,1 \cdot 15$ & 0.45 \\
\hline Multivar model $1 \dagger$ & 1 & Ref & 1.05 & $1 \cdot 00,1 \cdot 11$ & 1.06 & $1.00,1.13$ & 0.96 & $0.91,1.02$ & 1.01 & $0.94,1.07$ & 0.19 \\
\hline Multivar model $2 \ddagger$ & 1 & Ref & 1.01 & $0.96,1.07$ & 1.04 & $0.98,1.11$ & 0.95 & $0.89,1.02$ & 0.96 & $0.88,1.05$ & 0.09 \\
\hline Multivar model $3 \S$ & 1 & Ref & 1.01 & $0.96,1.07$ & 1.04 & $0.98,1.12$ & 0.95 & $0.89,1.02$ & 0.96 & $0.88,1.05$ & $0 \cdot 11$ \\
\hline \multicolumn{12}{|l|}{ CVD } \\
\hline Deaths $(n)$ & \multicolumn{2}{|c|}{823} & \multicolumn{2}{|c|}{1058} & \multicolumn{2}{|c|}{392} & \multicolumn{2}{|c|}{781} & \multicolumn{2}{|c|}{816} & \\
\hline Age-adjusted ${ }^{\star}$ & 1 & Ref & 1.09 & $0.99,1.21$ & $1 \cdot 17$ & $1 \cdot 05,1.30$ & 1.03 & $0.93,1.15$ & 1.09 & $0.98,1.22$ & 0.40 \\
\hline Multivar model $1 \dagger$ & 1 & Ref & 1.03 & $0.93,1.14$ & 1.09 & $0.98,1.21$ & 0.99 & $0.89,1.10$ & 1.01 & $0.91,1.13$ & 0.85 \\
\hline Multivar model $2 \ddagger$ & 1 & Ref & 0.99 & $0.89,1.09$ & 1.06 & $0.94,1.19$ & 0.97 & $0.86,1 \cdot 10$ & 0.96 & $0.82,1 \cdot 12$ & 0.48 \\
\hline Multivar model $3 \S$ & 1 & Ref & 0.99 & $0.90,1.10$ & 1.08 & $0.96,1.21$ & 1.00 & $0.88,1.13$ & 1.00 & $0.85,1.18$ & 0.96 \\
\hline \multicolumn{12}{|l|}{$\mathrm{CHD}$} \\
\hline Deaths $(n)$ & \multicolumn{2}{|c|}{505} & & 636 & & 230 & & 478 & & 534 & \\
\hline Age-adjusted ${ }^{*}$ & 1 & Ref & $1 \cdot 12$ & $0.99,1.27$ & $1 \cdot 19$ & $1.03,1.36$ & $1 \cdot 10$ & $0.96,1.26$ & 1.15 & $1.01,1.32$ & 0.16 \\
\hline Multivar model $1 \dagger$ & 1 & Ref & 1.06 & $0.93,1.20$ & $1 \cdot 12$ & $0.97,1.28$ & 1.07 & $0.93,1.22$ & 1.07 & $0.93,1.22$ & 0.58 \\
\hline Multivar model $2 \ddagger$ & 1 & Ref & 1.05 & $0.92,1.19$ & $1 \cdot 16$ & $1.00,1.35$ & $1 \cdot 15$ & $0.98,1.35$ & $1 \cdot 18$ & $0.97,1.44$ & 0.14 \\
\hline Multivar model $3 \S$ & 1 & Ref & 1.05 & $0.92,1.20$ & $1 \cdot 18$ & $1.02,1.37$ & $1 \cdot 18$ & $1.00,1.39$ & 1.23 & $1.00,1.50$ & 0.05 \\
\hline Cerebrovascular dise & & & & & & & & & & & \\
\hline Deaths $(n)$ & & 68 & & 198 & & 78 & & 148 & & 140 & \\
\hline Age-adjusted $^{*}$ & 1 & Ref & 0.97 & $0.78,1.21$ & 1.00 & $0.79,1.27$ & 0.84 & $0.66,1.06$ & 0.93 & $0.72,1.19$ & 0.39 \\
\hline Multivar model $1 \dagger$ & 1 & Ref & 0.92 & $0.74,1.14$ & 0.92 & $0.73,1.17$ & 0.79 & $0.62,1.00$ & 0.85 & $0.66,1.09$ & 0.16 \\
\hline Multivar model $2 \ddagger$ & 1 & Ref & 0.85 & $0.68,1.06$ & 0.80 & $0.62,1.03$ & 0.65 & $0.49,0.86$ & 0.60 & $0.42,0.86$ & 0.003 \\
\hline Multivar model $3 \S$ & 1 & Ref & 0.86 & $0.69,1.07$ & 0.82 & $0.63,1.06$ & 0.68 & $0.51,0.90$ & 0.65 & $0.45,0.94$ & 0.02 \\
\hline Sudden death & & & & & & & & & & & \\
\hline Deaths $(n)$ & & 55 & & 80 & & 16 & & 45 & & 47 & \\
\hline Age-adjusted* & 1 & Ref & 1.42 & $0.97,2.08$ & 1.07 & $0.69,1.68$ & 0.92 & $0.60,1.43$ & $1 \cdot 13$ & $0.74,1.74$ & 0.51 \\
\hline Multivar model $1 \dagger$ & 1 & Ref & 1.37 & $0.93,2.01$ & 1.03 & $0.66,1.61$ & 0.90 & $0.58,1.38$ & 1.06 & $0.69,1.62$ & 0.34 \\
\hline Multivar model $2 \ddagger$ & 1 & Ref & 1.38 & $0.93,2.04$ & 1.06 & $0.66,1.72$ & 0.94 & $0.56,1.57$ & $1 \cdot 14$ & $0.61,2.13$ & 0.87 \\
\hline Multivar model $3 \S$ & 1 & Ref & 1.38 & $0.93,2.04$ & 1.06 & $0.66,1.72$ & 0.94 & $0.56,1.58$ & 1.15 & $0 \cdot 61,2 \cdot 16$ & 0.89 \\
\hline
\end{tabular}

$\mathrm{E} \%$, energy percentage.

${ }^{*} \mathrm{HR}$ based on a stratified Cox model (year of birth: <1930, 1930-4, 1935-9, 1940-4, $\geq 1945$ ) with attained age as the time variable and with adjustment for sex.

† Model 1: age-adjusted model with additional adjustment for energy intake, systolic blood pressure, BMI, smoking and education.

¥ Model 2: model 1 with additional adjustment for intakes of SFA, ruminant TFA (rTFA), TFA from partially hydrogenated fish oil (PHFO), unsaturated cis-fatty acids, protein (all in $\mathrm{E} \%$ ) and cholesterol $(\mathrm{mg} / \mathrm{kJ}$ ).

$\S$ Model 3: model 1 with additional adjustment for intakes of SFA, rTFA, TFA from PHFO, carbohydrates, protein (all in E\%) and cholesterol (mg/kJ).

(HR comparing highest to lowest category of intake was 1.32, $95 \%$ CI 1.04, 1.69), although no significant trend was seen. The association was not significant with models 2 and 3. The associations did not differ for men and women (model 3: $P_{\text {interaction }} \geq 0 \cdot 12$ ). We found no significant interactions between intake of TFA from PHFO and BMI, except for death from all causes (with model 3: $P_{\text {interaction }}=0.004$, HR per $0.5 \mathrm{E} \%$ increase: $0.97(95 \%$ CI $0.95,0.99)$ and 1.00 (95\% CI $0.98,1.02$ ) for BMI $<25$ and $\geq 25 \mathrm{~kg} / \mathrm{m}^{2}$, respectively). We found significant interactions between intake of TFA from PHFO and follow-up for CVD (with model 3: $P_{\text {interaction }}=0.01, \mathrm{HR}$ per $0.5 \mathrm{E} \%$ increase: 0.96 (95\% CI 0.92 , $1.01)$ and 1.01 (95\% CI 0.98, 1.05) for follow-up $<10$ years and $\geq 10$ years after last measurement of intake, respectively) and for CHD (with model 3: $P_{\text {interaction }}=0.02$, HR per $0.5 \mathrm{E} \%$ increase $0.99(95 \%$ CI $0.93,1.04)$ and $1.04(95 \%$ CI $0.99,1.09)$ for follow-up $<10$ and $\geq 10$ years after last measurement of intake, respectively). Model 2 gave similar results on interaction.

\section{Ruminant trans-fatty acids}

Mean baseline intake of rTFA was $0.6 \mathrm{E} \%$. Men were in the majority in the highest category of rTFA intake (Table 5). With increasing category of rTFA intake, mean intakes of TFA from both PHVO and PHFO decreased, mean intakes of total fat, SFA and monounsaturated cis-fatty acids increased, whereas mean intakes of polyunsaturated cis-fatty acids and carbohydrates decreased. Serum cholesterol and TAG increased with increasing rTFA intake. The proportions of daily smokers increased, and the proportion of inactive subjects and subjects with higher education decreased, with increasing intake of rTFA. For most of the variables, the associations with rTFA intake were similar for men and women (data not shown), but we observed a slightly stronger association between rTFA intake and serum cholesterol for women than for men. Mean serum cholesterol in lowest and highest category of intake was 5.91 and $6.09 \mathrm{mmol} / 1$, respectively, for women and 6.16 and $6.21 \mathrm{mmol} / \mathrm{l}$, respectively, for men. 
Table 4. Hazard ratio (HR) and $95 \% \mathrm{Cl}$ of cause-specific death according to intake of trans-fatty acids (TFA) from partially hydrogenated fish oil (PHFO) (Number of deaths, hazard ratios and $95 \%$ confidence intervals)

\begin{tabular}{|c|c|c|c|c|c|c|c|c|c|c|c|}
\hline & \multicolumn{11}{|c|}{ Intake of TFA from PHFO (E\%) } \\
\hline & \multicolumn{2}{|c|}{$<0.85$} & \multicolumn{2}{|c|}{$0.85-1.349$} & \multicolumn{2}{|c|}{$1.35-1.849$} & \multicolumn{2}{|c|}{$1 \cdot 85-2 \cdot 349$} & \multicolumn{2}{|c|}{$\geq 2 \cdot 35$} & \multirow[b]{2}{*}{$P$} \\
\hline & $\mathrm{HR}$ & $95 \% \mathrm{Cl}$ & $\mathrm{HR}$ & $95 \% \mathrm{Cl}$ & $\mathrm{HR}$ & $95 \% \mathrm{Cl}$ & $\mathrm{HR}$ & $95 \% \mathrm{Cl}$ & $\mathrm{HR}$ & $95 \% \mathrm{Cl}$ & \\
\hline Person years & \multicolumn{2}{|c|}{419416} & \multicolumn{2}{|c|}{481339} & \multicolumn{2}{|c|}{355075} & \multicolumn{2}{|c|}{221887} & \multicolumn{2}{|c|}{365992} & \\
\hline \multicolumn{12}{|l|}{ All causes } \\
\hline Deaths $(n)$ & \multicolumn{2}{|c|}{2755} & \multicolumn{2}{|c|}{3077} & \multicolumn{2}{|c|}{2147} & \multicolumn{2}{|c|}{1355} & \multicolumn{2}{|c|}{2556} & \\
\hline Age-adjusted ${ }^{*}$ & 1 & Ref & 0.99 & $0.94,1.04$ & 0.95 & $0.90,1.01$ & 0.93 & $0.87,0.99$ & 1.09 & $1.03,1 \cdot 16$ & 0.002 \\
\hline Multivar model $1 \dagger$ & 1 & Ref & 1.02 & $0.96,1.07$ & 1.01 & $0.96,1.07$ & 0.98 & $0.91,1.04$ & $1 \cdot 10$ & $1.03,1 \cdot 16$ & 0.01 \\
\hline Multivar model $2 \ddagger$ & 1 & Ref & 1.01 & $0.95,1.06$ & 0.99 & $0.93,1.06$ & 0.94 & $0.87,1.02$ & 1.03 & $0.94,1.14$ & 0.40 \\
\hline Multivar model $3 \S$ & 1 & Ref & 1.00 & $0.95,1.05$ & 0.98 & $0.92,1.04$ & 0.93 & $0.86,1.00$ & 1.00 & $0.92,1.10$ & $0 \cdot 11$ \\
\hline \multicolumn{12}{|l|}{ CVD } \\
\hline Deaths $(n)$ & \multicolumn{2}{|c|}{912} & \multicolumn{2}{|c|}{1012} & \multicolumn{2}{|r|}{684} & \multicolumn{2}{|r|}{419} & \multicolumn{2}{|r|}{843} & \\
\hline Age-adjusted $^{*}$ & 1 & Ref & 1.00 & $0.92,1 \cdot 10$ & 0.96 & $0.87,1.06$ & 0.96 & $0.86,1.08$ & $1 \cdot 14$ & $1.03,1.27$ & 0.02 \\
\hline Multivar model $1 \dagger$ & 1 & Ref & 1.03 & $0.94,1 \cdot 13$ & 1.03 & $0.93,1.13$ & 1.02 & $0.90,1 \cdot 14$ & $1 \cdot 14$ & $1 \cdot 03,1 \cdot 26$ & 0.05 \\
\hline Multivar model $2 \ddagger$ & 1 & Ref & 1.04 & $0.94,1.14$ & 1.03 & $0.92,1.16$ & 1.03 & $0.89,1 \cdot 18$ & $1 \cdot 16$ & $0.98,1.38$ & 0.41 \\
\hline Multivar model $3 \S$ & 1 & Ref & 1.02 & $0.93,1.12$ & 1.00 & $0.90,1.12$ & 0.99 & $0.86,1 \cdot 13$ & 1.09 & $0.93,1.28$ & 0.89 \\
\hline \multicolumn{12}{|l|}{$\mathrm{CHD}$} \\
\hline Deaths $(n)$ & \multicolumn{2}{|c|}{601} & & 22 & & 406 & & 259 & & 495 & \\
\hline Age-adjusted $^{\star}$ & 1 & Ref & 0.98 & $0 \cdot 88,1 \cdot 10$ & 0.92 & $0.81,1.04$ & 0.92 & $0.79,1.07$ & 1.08 & $0.95,1.23$ & $0 \cdot 17$ \\
\hline Multivar model $1 \dagger$ & 1 & Ref & 1.01 & $0.91,1.14$ & 0.99 & $0.87,1.12$ & 0.97 & $0.84,1 \cdot 13$ & 1.07 & $0.94,1.22$ & 0.35 \\
\hline Multivar model $2 \ddagger$ & 1 & Ref & 1.02 & $0.91,1.15$ & 1.01 & $0.87,1.16$ & 1.00 & $0.84,1 \cdot 20$ & $1 \cdot 14$ & $0.91,1.41$ & $0 \cdot 16$ \\
\hline Multivar model 3§ & 1 & Ref & 1.01 & $0.90,1.14$ & 0.99 & $0.86,1.14$ & 0.98 & $0.82,1 \cdot 17$ & $1 \cdot 10$ & $0.89,1.35$ & 0.32 \\
\hline Cerebrovascular dise & & & & & & & & & & & \\
\hline Deaths $(n)$ & & 52 & & 191 & & 129 & & 89 & & 171 & \\
\hline Age-adjusted* ${ }^{\star}$ & 1 & Ref & $1 \cdot 10$ & $0.88,1.38$ & 1.02 & $0.81,1 \cdot 30$ & 1.07 & $0.82,1.40$ & $1 \cdot 30$ & $1.02,1.66$ & 0.22 \\
\hline Multivar model $1 \dagger$ & 1 & Ref & $1 \cdot 14$ & $0.91,1.42$ & 1.09 & $0.86,1.38$ & $1 \cdot 13$ & $0.87,1.48$ & $1 \cdot 32$ & $1.04,1.69$ & 0.23 \\
\hline Multivar model 2‡ & 1 & Ref & $1 \cdot 17$ & $0.93,1.47$ & 1.14 & $0.87,1.49$ & 1.20 & $0.87,1.66$ & 1.44 & $0.98,2 \cdot 12$ & 0.93 \\
\hline Multivar model $3 \S$ & 1 & Ref & $1 \cdot 13$ & $0.90,1.41$ & 1.06 & $0.82,1.37$ & 1.08 & $0.79,1.48$ & $1 \cdot 21$ & $0.84,1.76$ & 0.27 \\
\hline Sudden death & & & & & & & & & & & \\
\hline Deaths $(n)$ & & 52 & & 73 & & 41 & & 24 & & 53 & \\
\hline Age-adjusted $^{*}$ & 1 & Ref & 0.95 & $0.66,1.37$ & 1.09 & $0.74,1.59$ & 0.87 & $0.54,1.41$ & 1.07 & $0.71,1.62$ & 0.48 \\
\hline Multivar model $1 \dagger$ & 1 & Ref & 0.99 & $0.69,1.43$ & 1.18 & $0.80,1.73$ & 0.94 & $0.58,1.51$ & 1.08 & $0.71,1.63$ & 0.53 \\
\hline Multivar model $2 \ddagger$ & 1 & Ref & 0.96 & $0.66,1.39$ & 1.09 & $0.71,1.67$ & 0.83 & $0.47,1.45$ & 0.85 & $0.44,1.64$ & 0.84 \\
\hline Multivar model $3 \S$ & 1 & Ref & 0.96 & $0.66,1.40$ & $1 \cdot 11$ & $0.73,1.68$ & 0.85 & $0.49,1.46$ & 0.88 & $0.46,1.65$ & 0.93 \\
\hline
\end{tabular}

E\%, energy percentage.

${ }^{*} \mathrm{HR}$ based on a stratified Cox model (year of birth: <1930, 1930-4, 1935-9, 1940-4, $\geq 1945$ ) with attained age as the time variable and with adjustment for sex.

† Model 1: age-adjusted model with additional adjustment for energy intake, systolic blood pressure, BMI, smoking and education.

¥ Model 2: model 1 with additional adjustment for intakes of SFA, ruminant TFA (rTFA), TFA from partially hydrogenated vegetable oil (PHVO), unsaturated cis-fatty acids, protein (all in E\%) and cholesterol (mg/kJ).

$\S$ Model 3: model 1 with additional adjustment for intakes of SFA, rTFA, TFA from PHVO, carbohydrate, protein (all in E\%) and cholesterol (mg/kJ).

We found significant interactions between intake of rTFA and sex for risk of death from all causes, CVD, CHD and sudden death $\left(P_{\text {interaction }}=0.03,0.001,0.004\right.$ and 0.01, respectively, with model 3 ). Results are therefore presented separately for men and women.

In men, intake of rTFA was not associated with any of the cardiovascular causes of death with either of the multivariable models (Table 6). In women, intake was significantly, positively associated with risk of death from CVD, CHD and sudden death with model 1 , HR comparing highest to lowest category of intake were 1.30 (95\% CI 1.05, 1.61), $1.50(95 \%$ CI $1.11,2 \cdot 03)$ and 2.73 (95\% CI $1.19,6 \cdot 25)$, respectively (Table 7). With models 2 and 3 , these associations were somewhat attenuated and no longer significant. For sudden death, we found significant interactions between rTFA intake and BMI in women (with model 3: $P_{\text {interaction }}=0.02$, HR per $0.2 \mathrm{E} \%$ increase: $1.05(95 \%$ CI $0.29,3.77)$ and $1.72(95 \%$ CI $0.49,6 \cdot 11$ ) for $\mathrm{BMI}<25$ and $\geq 25 \mathrm{~kg} / \mathrm{m}^{2}$, respectively) and between rTFA intake and follow-up in men (with model 3: $P_{\text {interaction }}=0.0003$, HR per $0.2 \mathrm{E} \%$ increase: 0.49
(95\% CI $0.25,0.95)$ and 0.75 (95\% CI 0.39, 1.43) for follow-up $<10$ and $\geq 10$ years, respectively). For the other causes of death, the association did not differ with BMI or follow-up (model 3: $P_{\text {interaction }} \geq 0.08$ ). The interaction results were similar with model 2 .

\section{Discussion}

In the present prospective study, intake of TFA from PHVO was associated with increased risk of death from CHD and decreased risk of death from cerebrovascular diseases. Intake of TFA from PHFO was associated with increased risk of death from CVD and cerebrovascular diseases, but not with risk of $\mathrm{CHD}$ death. Intake of TFA from ruminant fat was positively associated with risk of death from CVD, CHD and sudden death in women, but not in men.

Previous prospective studies that have examined intake of industrially formed TFA and rTFA combined have found positive associations between intake of total TFA and $\operatorname{CHD}^{(9,14,15,24,25)}$. Most case-control studies have not been 
Table 5. Characteristics of study sample at baseline according to ruminant trans-fatty acids (rTFA) intake (Mean values and percentages)

\begin{tabular}{|c|c|c|c|c|c|}
\hline & \multicolumn{5}{|c|}{ rTFA intake at baseline (E\%) } \\
\hline & $<0.4$ & $0.4-0.549$ & $0.55-0.699$ & $0.7-0.849$ & $\geq 0.85$ \\
\hline Participants $(n)$ & 18891 & 20382 & 14263 & 8091 & 9837 \\
\hline Mean follow-up (years) & $25 \cdot 3$ & $25 \cdot 7$ & $26 \cdot 3$ & $26 \cdot 1$ & 25.9 \\
\hline Men (\%) & $49 \cdot 8$ & $47 \cdot 6$ & $50 \cdot 5$ & $47 \cdot 8$ & $58 \cdot 6$ \\
\hline Mean age at baseline (years) & $41 \cdot 2$ & $40 \cdot 8$ & $40 \cdot 3$ & $40 \cdot 6$ & 41.5 \\
\hline \multicolumn{6}{|l|}{ Baseline screening (\%) } \\
\hline Screening 1 & 33.0 & $38 \cdot 3$ & 43.4 & $45 \cdot 2$ & 48.4 \\
\hline Screening 2 & 28.4 & $28 \cdot 7$ & $31 \cdot 0$ & $28 \cdot 3$ & $25 \cdot 3$ \\
\hline Screening 3 & 38.5 & $32 \cdot 9$ & $25 \cdot 5$ & $26 \cdot 5$ & $26 \cdot 3$ \\
\hline Mean intake of TFA from PHVO (E\%) & $1 \cdot 14$ & 1.09 & 0.96 & 0.64 & 0.25 \\
\hline Mean intake of TFA from PHFO (E\%) & 1.72 & 1.71 & 1.66 & 1.48 & $1 \cdot 11$ \\
\hline Mean energy intake $(\mathrm{kJ} / \mathrm{d})$ & 6765 & 7011 & 7362 & 7021 & 7852 \\
\hline Mean total fat intake (E\%) & 35.7 & 37.5 & $39 \cdot 0$ & $39 \cdot 2$ & 41.4 \\
\hline Mean saturated fat intake (E\%) & $12 \cdot 1$ & $14 \cdot 1$ & $15 \cdot 9$ & $17 \cdot 4$ & $20 \cdot 8$ \\
\hline Mean monounsaturated cis-fatty acids intake (E\%) & $8 \cdot 6$ & $9 \cdot 1$ & $9 \cdot 6$ & $9 \cdot 8$ & $10 \cdot 5$ \\
\hline Mean polyunsaturated cis-fatty acids intake (E\%) & 6.9 & $6 \cdot 4$ & $5 \cdot 8$ & 4.7 & 3.5 \\
\hline Mean carbohydrate intake (E\%) & 48.5 & $46 \cdot 9$ & $45 \cdot 6$ & $45 \cdot 2$ & $43 \cdot 8$ \\
\hline Mean protein intake $(\mathrm{E} \%)$ & $15 \cdot 8$ & $15 \cdot 6$ & $15 \cdot 4$ & $15 \cdot 6$ & $14 \cdot 8$ \\
\hline Mean cholesterol intake (mg/MJ) & 33.7 & $35 \cdot 1$ & $37 \cdot 0$ & 39.5 & 41.9 \\
\hline Mean systolic blood pressure (mmHg) & 131 & 131 & 132 & 132 & 133 \\
\hline Mean total serum cholesterol ( $\mathrm{mmol} / \mathrm{l})$ & 6.03 & 6.05 & 6.06 & 6.08 & $6 \cdot 16$ \\
\hline Mean serum TAG (mmol/l) & 1.63 & 1.63 & 1.67 & 1.67 & 1.77 \\
\hline Daily smokers (\%) & 38.8 & 41.7 & $45 \cdot 7$ & $46 \cdot 2$ & $48 \cdot 3$ \\
\hline Mean BMI $\left(\mathrm{kg} / \mathrm{m}^{2}\right)$ & 24.9 & $24 \cdot 6$ & $24 \cdot 3$ & $24 \cdot 4$ & $24 \cdot 3$ \\
\hline Inactive (\%) & $26 \cdot 5$ & $25 \cdot 5$ & $23 \cdot 8$ & $22 \cdot 7$ & $21 \cdot 0$ \\
\hline Higher education (\%) & $16 \cdot 1$ & $14 \cdot 2$ & $12 \cdot 5$ & $12 \cdot 5$ & $12 \cdot 1$ \\
\hline
\end{tabular}

E\%, energy percentage; TFA, trans-fatty acids; PHVO, partially hydrogenated vegetable oil; PHFO, partially hydrogenated fish oil.

able to distinguish between different sources of TFA, as TFA in adipose tissue has been used as a marker of intake ${ }^{(26-29)}$. Positive associations between TFA in adipose tissue and CHD risk have been reported ${ }^{(27-29)}$, whereas the results from the EURAMIC study were more conflicting ${ }^{(26)}$. Two prospective studies ${ }^{(12,14)}$ and one case-control study ${ }^{(13)}$ have examined TFA intake from different sources. These studies found positive associations between intake of TFA from vegetable sources and risk of CHD that are in accordance with the present results on intake of TFA from PHVO and risk of CHD.

Considering the more unfavourable effect of PHFO than of PHVO on blood lipids, we expected to find a stronger association between intake and risk of CHD for PHFO than for PHVO. The opposite was observed, however. The reason for this is unclear. One explanation may be that TFA from PHVO has a more unfavourable effect on the fibrinolytic system. It has been shown that the postprandial level of tissue plasminogen activator was significantly lower on a PHVO diet than on a palm oil diet ${ }^{(30)}$. Furthermore, intake of PHVO has been shown to result in higher levels of plasminogen activator inhibitor type 1 antigen and activity compared to $\mathrm{PHFO}^{(31)}$. It was speculated that this more unfavourable haemostatic profile may be related to the much higher amount of 18:1 trans (elaidic acid and its isomers) in PHVO than in $\mathrm{PHFO}^{(31)}$. Furthermore, it has also been reported that circulating trans-palmitoleate is associated with lower insulin resistance, less dyslipidaemia and incident diabetes ${ }^{(32)}$. PHFO contains appreciable amounts of trans-palmitoleic acid $(16: 1 \text { trans })^{(4)}$. We cannot exclude that such metabolic effect to a certain extent may counteract the unfavourable effects of PHFO on blood cholesterol.

We found that intake of TFA from PHVO was associated with decreased risk of cerebrovascular diseases, whereas intake of TFA from PHFO was associated with increased risk. To our knowledge, these associations have not been studied previously. One possible explanation is that the inverse association is due to chance. However, a high serum cholesterol level may increase the risk of stroke ${ }^{(33,34)}$, and the different effects we found of TFA from PHVO and PHFO on serum cholesterol provide a possible explanation for the differing results on cerebrovascular risk. Intake of TFA from PHVO was not associated with total serum cholesterol (Table 1), whereas total serum cholesterol increased with increasing intake of TFA from PHFO (Table 2). PHVO was used in soft margarines based on vegetable oils that were high in cis MUFA and PUFA, whereas PHFO was primarily used in hard margarines and shortenings with higher contents of both trans and SFA and a smaller amount of unsaturated fatty acids. Intake of PHVO may also be associated with a more health conscious lifestyle that we have not been able to measure all aspects of, and some residual confounding can not be excluded for any of the studied associations.

Although trans isomers present in ruminant fat and PHVO are quite similar, TFA isomeric distribution may be very different $^{(35)}$. The trans isomers seem, however, to have essentially the same effects on plasma lipids ${ }^{(5,6)}$, and it is therefore to be expected that their health effects are similar. We found that intake of rTFA was significantly, positively associated with risk of death from CVD and CHD in women, but not 
Table 6. Hazard ratio (HR) and $95 \% \mathrm{Cl}$ of cause-specific death according to intake of ruminant trans-fatty acids (rTFA) (men)

(Number of deaths, hazard ratios and $95 \%$ confidence intervals)

\begin{tabular}{|c|c|c|c|c|c|c|c|c|c|c|c|}
\hline & \multicolumn{11}{|c|}{ Intake of rTFA (E\%) } \\
\hline & \multicolumn{2}{|c|}{$<0.4$} & \multicolumn{2}{|c|}{$0.4-0.549$} & \multicolumn{2}{|c|}{$0.55-0.699$} & \multicolumn{2}{|c|}{$0.7-0.849$} & \multicolumn{2}{|c|}{$\geq 0.85$} & \multirow[b]{2}{*}{$P$} \\
\hline & $\mathrm{HR}$ & $95 \% \mathrm{Cl}$ & $\mathrm{HR}$ & $95 \% \mathrm{Cl}$ & $\mathrm{HR}$ & $95 \% \mathrm{Cl}$ & $\mathrm{HR}$ & $95 \% \mathrm{Cl}$ & $\mathrm{HR}$ & $95 \% \mathrm{Cl}$ & \\
\hline Person years & \multicolumn{2}{|c|}{233009} & \multicolumn{2}{|c|}{244714} & \multicolumn{2}{|c|}{187053} & \multicolumn{2}{|c|}{98967} & \multicolumn{2}{|c|}{146851} & \\
\hline \multicolumn{12}{|l|}{ All causes } \\
\hline Deaths $(n)$ & \multicolumn{2}{|c|}{1826} & \multicolumn{2}{|r|}{1937} & \multicolumn{2}{|c|}{1564} & \multicolumn{2}{|c|}{867} & \multicolumn{2}{|c|}{1388} & \\
\hline Age-adjusted $^{*}$ & 1 & Ref & 1.03 & $0.97,1.10$ & $1 \cdot 12$ & $1.04,1 \cdot 20$ & $1 \cdot 13$ & $1.04,1 \cdot 22$ & $1 \cdot 11$ & $1.03,1.19$ & 0.001 \\
\hline Multivar model $1 \dagger$ & 1 & Ref & 1.03 & $0.96,1.10$ & 1.09 & $1.01,1 \cdot 16$ & 1.09 & $1.01,1 \cdot 18$ & 1.08 & $1.00,1.16$ & 0.03 \\
\hline Multivar model 2‡ & 1 & Ref & 1.00 & $0.93,1.07$ & 1.03 & $0.94,1.13$ & 1.03 & $0.91,1.16$ & 0.98 & $0.83,1.16$ & 0.03 \\
\hline Multivar model $3 \S$ & 1 & Ref & 1.00 & $0.93,1.08$ & 1.04 & $0.95,1.14$ & 1.03 & $0.91,1.17$ & 0.98 & $0.83,1.16$ & 0.02 \\
\hline \multicolumn{12}{|l|}{ CVD } \\
\hline Deaths $(n)$ & \multicolumn{2}{|c|}{715} & \multicolumn{2}{|r|}{754} & \multicolumn{2}{|r|}{580} & \multicolumn{2}{|r|}{312} & \multicolumn{2}{|r|}{481} & \\
\hline Age-adjusted $^{*}$ & 1 & Ref & 1.06 & $0.95,1.18$ & 1.07 & $0.95,1.19$ & 1.06 & $0.93,1.21$ & 0.99 & $0.88,1.12$ & 0.70 \\
\hline Multivar model $1 \dagger$ & 1 & Ref & 1.07 & $0.96,1 \cdot 18$ & 1.05 & $0.94,1 \cdot 18$ & 1.04 & $0.91,1 \cdot 18$ & 0.98 & $0.87,1 \cdot 11$ & 0.57 \\
\hline Multivar model $2 \ddagger$ & 1 & Ref & 1.05 & $0.93,1.18$ & 1.03 & $0.88,1 \cdot 20$ & 1.01 & $0.82,1.24$ & 0.94 & $0.71,1.26$ & 0.92 \\
\hline Multivar model 3§ & 1 & Ref & 1.04 & $0.92,1.17$ & 1.00 & $0.86,1 \cdot 17$ & 0.97 & $0.79,1 \cdot 19$ & 0.89 & $0.66,1.19$ & 0.26 \\
\hline \multicolumn{12}{|l|}{$\mathrm{CHD}$} \\
\hline Deaths $(n)$ & \multicolumn{2}{|c|}{460} & & 488 & & 400 & & 212 & & 326 & \\
\hline Age-adjusted $^{*}$ & 1 & Ref & 1.03 & $0.91,1.18$ & 1.06 & $0.93,1.22$ & $1 \cdot 11$ & $0.94,1.30$ & 1.00 & $0.86,1 \cdot 15$ & $0 \cdot 81$ \\
\hline Multivar model $1 \dagger$ & 1 & Ref & 1.04 & $0.91,1.18$ & 1.04 & $0.91,1.19$ & 1.08 & $0.92,1.27$ & 0.98 & $0.85,1.14$ & 0.96 \\
\hline Multivar model $2 \ddagger$ & 1 & Ref & 1.01 & $0.88,1 \cdot 17$ & 1.00 & $0 \cdot 83,1 \cdot 21$ & 1.04 & $0.80,1.34$ & 0.93 & $0.64,1.33$ & 0.49 \\
\hline Multivar model $3 \S$ & 1 & Ref & 1.00 & $0.86,1 \cdot 16$ & 0.97 & $0 \cdot 80,1 \cdot 18$ & 1.00 & $0.77,1 \cdot 29$ & 0.87 & $0.60,1.25$ & 0.92 \\
\hline Cerebrovascular dise & & & & & & & & & & & \\
\hline Deaths $(n)$ & & 21 & & 115 & & 88 & & 45 & & 73 & \\
\hline Age-adjusted* ${ }^{\star}$ & 1 & Ref & 1.08 & $0.83,1.39$ & 0.99 & $0.75,1.32$ & 0.84 & $0.59,1.20$ & 1.01 & $0.75,1.36$ & 0.85 \\
\hline Multivar model $1 \dagger$ & 1 & Ref & 1.08 & $0.83,1.40$ & 0.98 & $0.74,1.30$ & 0.81 & $0.57,1.16$ & 1.01 & $0.75,1.36$ & 0.79 \\
\hline Multivar model 2‡ & 1 & Ref & 1.05 & $0.78,1.40$ & 0.92 & $0.63,1.34$ & 0.72 & $0.43,1.20$ & 0.80 & $0.41,1.58$ & 0.89 \\
\hline Multivar model $3 \S$ & 1 & Ref & 1.02 & $0.76,1.36$ & 0.88 & $0.60,1 \cdot 28$ & 0.67 & $0.40,1 \cdot 11$ & 0.71 & $0.36,1.40$ & 0.33 \\
\hline Sudden death & & & & & & & & & & & \\
\hline Deaths $(n)$ & & 43 & & 42 & & 32 & & 36 & & 29 & \\
\hline Age-adjusted $^{*}$ & 1 & Ref & 0.98 & $0.65,1.48$ & 0.85 & $0.54,1.34$ & 1.57 & $1.00,2.47$ & 0.78 & $0.48,1.29$ & 0.87 \\
\hline Multivar model $1 \dagger$ & 1 & Ref & 0.97 & $0.64,1.46$ & 0.82 & $0.51,1.29$ & 1.49 & $0.95,2.35$ & 0.75 & $0.45,1.24$ & 0.71 \\
\hline Multivar model 2‡ & 1 & Ref & 0.89 & $0.56,1.42$ & 0.70 & $0.38,1.31$ & 1.23 & $0.57,2.66$ & 0.56 & $0.17,1.80$ & 0.33 \\
\hline Multivar model $3 \S$ & 1 & Ref & 0.90 & $0.56,1.43$ & 0.71 & $0.38,1.33$ & 1.24 & $0.57,2.70$ & 0.57 & $0 \cdot 18,1 \cdot 84$ & 0.35 \\
\hline
\end{tabular}

$\mathrm{E} \%$, energy percentage.

${ }^{*} \mathrm{HR}$ based on a stratified Cox model (year of birth: <1930, 1930-4, 1935-9, 1940-4, $\geq 1945$ ) with attained age as the time variable and with adjustment for sex and interaction between rTFA intake and sex.

† Model 1: age-adjusted model with additional adjustment for energy intake, systolic blood pressure, BMI, smoking and education.

$\ddagger$ Model 2: model 1 with additional adjustment for intakes of SFA, trans-fatty acids (TFA) from partially hydrogenated vegetable oil (PHVO), TFA from partially hydrogenated fish oil (PHFO), unsaturated cis-fatty acids, protein (all in E\%) and cholesterol (mg/kJ).

§ Model 3: model 1 with additional adjustment for intakes of SFA, TFA from PHVO, TFA from PHFO, carbohydrate, protein (all in E\%) and cholesterol (mg/kJ).

in men. The positive associations were significant only in model 1, not in models 2 and 3. Previous studies have found a significant inverse association ${ }^{(14)}$, a non-significant inverse association $^{(12)}$, no association ${ }^{(13,16)}$ and a weak, nonsignificant, positive association ${ }^{(15)}$ with CHD risk. Most of these studies ${ }^{(12,13,15,16)}$ have adjusted for intake of saturated fat. We also adjusted for saturated fat in models 2 and 3, but not in model 1. Intakes of saturated fat and rTFA will always be highly correlated because the sources of rTFA are also sources of saturated fat. In the present study, the Pearson correlation coefficient between intakes of saturated fat and rTFA at baseline was 0.89. It is therefore possible that the positive associations that we observed with model 1 are caused by saturated fat, not rTFA. This is not likely, however, because the associations were still positive with further adjustment for saturated fat, even though they were weaker and no longer significant. Inclusion of intake of both rTFA and saturated fat in the same multivariable model may lead to over adjustment. This may explain why the associations were no longer significant and why no positive association has previously been found. Furthermore, it makes interpretation of the results difficult, as was pointed out by Mozaffarian ${ }^{(36)}$ in a commentary to the study by Jakobsen et al. ${ }^{(16)}$.

Most previous prospective studies have included only one $\operatorname{sex}^{(12,14,15,24,25)}$ and have not been able to study sex differences. Except for the EURAMIC study ${ }^{(26)}$, case-control studies have usually included both men and women ${ }^{(13,27-29)}$, and effect estimates have been reported for men and women combined $^{(27-29)}$. However, Baylin et al. ${ }^{(28)}$ found no significant interaction between TFA and sex, and Ascherio et al. ${ }^{(13)}$ found similar associations between intake of TFA and risk of CHD in men and women. This is in accordance with the present results, as we did not find evidence of interaction between sex and intake of industrially formed TFA. However, we found significant interactions between intake of rTFA and sex, in contrast with the study by Jakobsen et al. ${ }^{(16)}$, in which no significant interactions between sex and rTFA intake were found. We found a stronger association between rTFA intake and serum cholesterol among women than among men. 
Table 7. Hazard ratio (HR) and $95 \% \mathrm{Cl}$ of cause-specific death according to intake of ruminant trans-fatty acid (rTFA) (women) (Number of deaths, hazard ratios and $95 \%$ confidence intervals)

\begin{tabular}{|c|c|c|c|c|c|c|c|c|c|c|c|}
\hline & \multicolumn{11}{|c|}{ Intake of rTFA (E\%) } \\
\hline & \multicolumn{2}{|c|}{$<0.4$} & \multicolumn{2}{|c|}{$0.4-0.549$} & \multicolumn{2}{|c|}{$0.55-0.699$} & \multicolumn{2}{|c|}{$0.7-0.849$} & \multicolumn{2}{|c|}{$\geq 0.85$} & \multirow[b]{2}{*}{$P$} \\
\hline & $\mathrm{HR}$ & $95 \% \mathrm{Cl}$ & $\mathrm{HR}$ & $95 \% \mathrm{Cl}$ & $\mathrm{HR}$ & $95 \% \mathrm{Cl}$ & $\mathrm{HR}$ & $95 \% \mathrm{Cl}$ & $\mathrm{HR}$ & $95 \% \mathrm{Cl}$ & \\
\hline Person years & \multicolumn{2}{|c|}{244156} & \multicolumn{2}{|c|}{280033} & \multicolumn{2}{|c|}{188560} & \multicolumn{2}{|c|}{112499} & \multicolumn{2}{|c|}{107867} & \\
\hline \multicolumn{12}{|l|}{ All causes } \\
\hline Deaths $(n)$ & \multicolumn{2}{|c|}{1058} & \multicolumn{2}{|r|}{1230} & \multicolumn{2}{|c|}{869} & \multicolumn{2}{|r|}{560} & \multicolumn{2}{|r|}{591} & \\
\hline Age-adjusted* & 1 & Ref & 0.99 & $0.91,1.07$ & $1 \cdot 10$ & $1.01,1.21$ & $1 \cdot 10$ & $0.99,1.22$ & 1.24 & $1 \cdot 11,1.38$ & $<0.001$ \\
\hline Multivar model $1 \dagger$ & 1 & Ref & 0.97 & $0.89,1.05$ & 1.06 & $0.97,1.16$ & 1.04 & $0.94,1.16$ & $1 \cdot 19$ & $1.07,1.33$ & $<0.001$ \\
\hline Multivar model $2 \ddagger$ & 1 & Ref & 0.95 & $0.87,1.03$ & 1.02 & $0.92,1.13$ & 0.99 & $0.86,1.13$ & $1 \cdot 10$ & $0.93,1.31$ & 0.17 \\
\hline Multivar model $3 \S$ & 1 & Ref & 0.95 & $0.87,1.04$ & 1.02 & $0.92,1.14$ & 0.99 & $0.87,1.14$ & $1 \cdot 11$ & $0.93,1.31$ & 0.15 \\
\hline \multicolumn{12}{|l|}{ CVD } \\
\hline Deaths $(n)$ & \multicolumn{2}{|c|}{265} & \multicolumn{2}{|r|}{267} & \multicolumn{2}{|c|}{199} & \multicolumn{2}{|c|}{145} & \multicolumn{2}{|c|}{152} & \\
\hline Age-adjusted ${ }^{\star}$ & 1 & Ref & 0.92 & $0.78,1.10$ & 0.98 & $0.81,1 \cdot 18$ & $1 \cdot 11$ & $0.90,1.37$ & 1.30 & $1 \cdot 05,1 \cdot 61$ & $<0.001$ \\
\hline Multivar model $1 \dagger$ & 1 & Ref & 0.90 & $0.76,1.07$ & 0.95 & $0.79,1.14$ & 1.06 & $0.86,1.31$ & 1.30 & $1.05,1.61$ & 0.002 \\
\hline Multivar model $2 \ddagger$ & 1 & Ref & 0.90 & $0.75,1.07$ & 0.93 & $0.75,1.15$ & 1.04 & $0.80,1.34$ & 1.26 & $0.92,1.73$ & 0.27 \\
\hline Multivar model $3 \S$ & 1 & Ref & 0.89 & $0.74,1.06$ & 0.91 & $0.74,1 \cdot 12$ & 1.00 & $0.77,1.30$ & 1.20 & $0.88,1.65$ & 0.91 \\
\hline \multicolumn{12}{|l|}{$\mathrm{CHD}$} \\
\hline Deaths $(n)$ & \multicolumn{2}{|c|}{120} & & 135 & & 87 & & 74 & & 81 & \\
\hline Age-adjusted ${ }^{*}$ & 1 & Ref & 0.99 & $0.77,1.27$ & 0.93 & $0.71,1.23$ & $1 \cdot 24$ & $0.92,1.68$ & 1.50 & $1.11,2.03$ & 0.001 \\
\hline Multivar model $1 \dagger$ & 1 & Ref & 0.97 & $0.75,1.24$ & 0.91 & $0.69,1.19$ & 1.19 & $0.88,1.60$ & 1.50 & $1.11,2.03$ & 0.001 \\
\hline Multivar model $2 \ddagger$ & 1 & Ref & 0.95 & $0.73,1.23$ & 0.88 & $0.65,1.19$ & $1 \cdot 14$ & $0.80,1.62$ & 1.42 & $0.94,2.16$ & 0.07 \\
\hline Multivar model $3 \S$ & 1 & Ref & 0.94 & $0.73,1.21$ & 0.86 & $0.63,1.16$ & $1 \cdot 10$ & $0.77,1.56$ & 1.35 & $0.89,2.05$ & 0.30 \\
\hline Cerebrovascular dise & & & & & & & & & & & \\
\hline Deaths $(n)$ & & 84 & & 68 & & 60 & & 44 & & 34 & \\
\hline Age-adjusted $^{*}$ & 1 & Ref & 0.86 & $0.63,1.19$ & 1.00 & $0.72,1.41$ & 1.02 & $0.68,1.51$ & $1 \cdot 11$ & $0.73,1.67$ & 0.36 \\
\hline Multivar model $1 \dagger$ & 1 & Ref & 0.84 & $0.61,1.15$ & 0.95 & $0.68,1.34$ & 0.96 & $0.64,1.42$ & 1.08 & $0.71,1.63$ & 0.49 \\
\hline Multivar model $2 \ddagger$ & 1 & Ref & 0.81 & $0.58,1.14$ & 0.89 & $0.59,1.34$ & 0.85 & $0.50,1.45$ & 0.89 & $0.45,1.75$ & 0.89 \\
\hline Multivar model $3 \S$ & 1 & Ref & 0.80 & $0.57,1.12$ & 0.86 & $0.57,1.29$ & 0.80 & $0.47,1.36$ & 0.81 & $0.41,1.59$ & 0.51 \\
\hline Sudden death & & & & & & & & & & & \\
\hline Deaths $(n)$ & & 13 & & 14 & & 13 & & 7 & & 14 & \\
\hline Age-adjusted* & 1 & Ref & 1.00 & $0.45,2.26$ & 1.50 & $0.66,3.37$ & 1.86 & $0.78,4.48$ & 2.97 & $1 \cdot 30,6 \cdot 78$ & 0.004 \\
\hline Multivar model $1 \dagger$ & 1 & Ref & 0.97 & $0.43,2.18$ & 1.40 & $0.62,3 \cdot 15$ & 1.72 & $0.71,4.13$ & 2.73 & $1 \cdot 19,6 \cdot 25$ & 0.01 \\
\hline Multivar model $2 \ddagger$ & 1 & Ref & 0.92 & $0.40,2.12$ & 1.26 & $0.51,3.10$ & 1.48 & $0.51,4.28$ & $2 \cdot 19$ & $0.63,7.58$ & 0.96 \\
\hline Multivar model $3 \S$ & 1 & Ref & 0.92 & $0.40,2.13$ & 1.27 & $0.51,3.13$ & 1.49 & $0.51,4.32$ & 2.21 & $0.63,7.72$ & 0.95 \\
\hline
\end{tabular}

E\%, energy percentage.

${ }^{*}$ HR based on a stratified Cox model (year of birth: $<1930,1930-4,1935-9,1940-4, \geq 1945$ ) with attained age as the time variable and with adjustment for sex and interaction between rTFA intake and sex.

† Model 1: age-adjusted model with additional adjustment for energy intake, systolic blood pressure, BMI, smoking and education.

$\ddagger$ Model 2: model 1 with additional adjustment for intakes of SFA, trans-fatty acids (TFA) from partially hydrogenated vegetable oil (PHVO), TFA from partially hydrogenated fish oil (PHFO), unsaturated cis-fatty acids, protein (all in E\%) and cholesterol (mg/kJ).

$\S$ Model 3: model 1 with additional adjustment for intakes of SFA, TFA from PHVO, TFA from PHFO, carbohydrate, protein (all in E\%) and cholesterol (mg/kJ).

The effects of rTFA on LDL- and total cholesterol have been found to be more unfavourable for women ${ }^{(5)}$.

Indication of effect modification by BMI has been found previously ${ }^{(24)}$. The present results do not indicate that the associations between TFA intake and CVD or CHD risk differ for BMI $<25$ and $\geq 25 \mathrm{~kg} / \mathrm{m}^{2}$. We found, however, a significant interaction between BMI and intake of TFA from PHFO for all causes of death, but the effect estimates found for the two BMI categories were quite similar. We also found a significant interaction between BMI and rTFA intake for sudden death in women, but the associations were non-significant for both BMI categories. We found significant interactions between TFA intake and follow-up time. For TFA from PHVO, the associations with CVD and CHD were stronger during the first period of follow-up, and we found no associations $\geq 10$ years after the last measurement. This can probably be explained by the considerable reduction in the intake of industrial TFA that occurred in Norway during the last period of follow-up (1997-2007), as margarine has been essentially trans-free after 1997. However, the association between intake of TFA from PHFO and risk of CHD was stronger $\geq 10$ years after the last measurement than earlier. Thus, the effects of PHFO may be more long-term than the effects of TFA from PHVO. For rTFA, the association did not differ for follow-up $<10$ and $\geq 10$ years since last measurement, except for sudden death in men.

We explored several multivariable models. We expected to see stronger associations when comparing TFA intake with one specific nutrient (models 2 and 3) than with a combination of all other energy sources (model 1). This was the case with TFA from PHVO, in which intake was significantly associated with risk of death from CHD only when compared to intake of unsaturated cis-fatty acids and with risk of cerebrovascular diseases when compared to either intake of unsaturated cis-fatty acids or carbohydrates. In contrast, for TFA from PHFO and ruminant fat, the significant associations were found when comparing intake with all other energy sources. However, the estimated HR from the different multivariable models were quite similar. 
Strengths of the present study include the prospective design with participants from the general population and a very high attendance. The present study is the first prospective study to include both men and women; thus, we have been able to study sex differences. Furthermore, the FFQ was designed specifically to measure fat intake in the Norwegian diet, and we have accurate information from the margarine industry on the compositions of the different types of margarine at the time of the screenings. Intake of industrially formed TFA in Norway has decreased substantially, and because we have up to three measurements of the participants' intake, we have taken at least some of the changes that have occurred into account. However, intake was not measured after 1988. This may have influenced the results, as we found evidence that the associations between TFA intake and risk differed for follow-up time $<10$ and $\geq 10$ years after last measurement. The end points in the present study were obtained from the Cause of Death Registry. All deaths in Norway are reported to the Cause of Death Registry. The linkage was secured by using the personal identification numbers. Thus, total mortality is a valid end point. The percentage of deaths that are autopsied has gradually decreased over time in Norway, and the cause of death relies to a great extent on physicians' certificates. Misclassification of cause of death may occur. It is not likely, however, that the problem of misclassification of cause of death depends on intake of TFA.

In conclusion, the present study confirms that intake of TFA from $\mathrm{PHVO}$ is positively associated with increased risk of CHD. Furthermore, intake of TFA from PHFO was associated with increased risk of CVD and cerebrovascular diseases, but not with risk of CHD. New in the present study is the finding that intake of TFA from ruminant fat seems to increase risk of CVD and CHD in women, but not in men. The negative association between TFA from PHVO and cerebrovascular disease warrants further investigation.

\section{Acknowledgements}

The present study received funding from the Throne-Holst Foundation for Nutrition Research. R. S., A. S. L. and A. T. contributed to data collection. J. I. P., R. S., B. K., A. S. L. and A. T. provided essential material. I. L. and M. B. V. performed the analyses. I. L., M. B. V. and J. I. P. initiated the present study and wrote the paper. I. L. had primary responsibility for the final content. All authors contributed to the design of the research and interpretation of the data. All authors revised the manuscript critically for important intellectual content and read and approved the final manuscript. We thank all the participants in the Norwegian Counties Study and the professional staff who made the present study possible, including those of the former Norwegian Health Screening Service (now part of the Norwegian Institute of Public Health), who initiated the Norwegian Counties Study in 1974 and continued data collection over the following years. We also thank Mills DA, Norway, for providing information on the composition of margarines and fats and Thor Kristoffersen, former director of A/S Denofa og Lilleborg Fabrikker, for information on the hydrogenation conditions used for partially hydrogenation of soyabean oil and fish oils. J. I. P. is a member of the scientific advisory board of the food company Mills DA, Oslo. The remaining authors declare no conflict of interest.

\section{References}

1. Mozaffarian D, Katan MB, Ascherio A, et al. (2006) Medical progress - trans-fatty acids and cardiovascular disease. $N$ Engl J Med 354, 1601-1613.

2. Anonymous (2009) WHO Scientific Update on health consequences of trans fatty acids. Eur J Clin Nutr 63, Suppl. 2, S1-S75.

3. Mensink RP \& Katan MB (1990) Effect of dietary trans-fattyacids on high-density and low-density-lipoprotein cholesterol levels in healthy-subjects. $N$ Engl J Med 323, 439-445.

4. Almendingen K, Jordal O, Kierulf $\mathrm{P}$, et al. (1995) Effects of partially hydrogenated fish-oil, partially hydrogenated soybean oil, and butter on serum-lipoproteins and Lp[A] in men. J Lipid Res 36, 1370-1384.

5. Chardigny JM, Destaillats F, Malpuech-Brugere C, et al. (2008) Do trans fatty acids from industrially produced sources and from natural sources have the same effect on cardiovascular disease risk factors in healthy subjects? Results of the trans Fatty Acids Collaboration (TRANSFACT) study. Am J Clin Nutr 87, 558-566.

6. Motard-Belanger A, Charest A, Grenier G, et al. (2008) Study of the effect of trans fatty acids from ruminants on blood lipids and other risk factors for cardiovascular disease. Am J Clin Nutr 87, 593-599.

7. Ascherio A, Katan MB, Zock PL, et al. (1999) Trans fatty acids and coronary heart disease. $N$ Engl J Med $\mathbf{3 4 0}$, 1994-1998.

8. Mozaffarian D \& Willett WC (2007) Trans fatty acids and cardiovascular risk: a unique cardiometabolic imprint? Curr Atheroscler Rep 9, 486-493.

9. Hu FB, Stampfer MJ, Manson JE, et al. (1997) Dietary fat intake and the risk of coronary heart disease in women. $N$ Engl J Med 337, 1491-1499.

10. Opstvedt J, Urdahl N \& Pettersen J (1990) Fish oils - an old fat source with new possibilities. In Edible Fats and Oils Processing: Basic Principles and Modern Practices: World Conference Proceedings, pp. 250-259. Champaign, Illinois.

11. Ministry of Agriculture and Food (1960) Report from the Margarine Control. Oslo: Ministry of Agriculture and Food.

12. Willett WC, Stampfer MJ, Manson JE, et al. (1993) Intake of trans-fatty-acids and risk of coronary heart-disease among women. Lancet 341, 581-585.

13. Ascherio A, Hennekens CH, Buring JE, et al. (1994) Trans-fatty-acids intake and risk of myocardial-infarction. Circulation 89, 94-101.

14. Pietinen P, Ascherio A, Korhonen P, et al. (1997) Intake of fatty acids and risk of coronary heart disease in a cohort of Finnish men - the Alpha-Tocopherol, Beta-Carotene Cancer Prevention Study. Am J Epidemiol 145, 876-887.

15. Oomen CM, Ocke MC, Feskens EJM, et al. (2001) Association between trans fatty acid intake and 10-year risk of coronary heart disease in the Zutphen Elderly Study: a prospective population-based study. Lancet 357, 746-751.

16. Jakobsen MU, Overvad K, Dyerberg J, et al. (2008) Intake of ruminant trans fatty acids and risk of coronary heart disease. Int J Epidemiol 37, 173-182.

17. Bjartveit K, Foss OP, Gjervig T, et al. (1979) The cardio vascular disease study in Norwegian Counties. Background and organization. Acta Med Scand Suppl 634, 1-70. 
18. Gaard M, Tretli S \& Loken EB (1995) Dietary fat and the risk of breast cancer: a prospective study of 25,892 Norwegian women. Int J Cancer 63, 13-17.

19. Hulshof KFAM, van Erp-Baart MA, Anttolainen M, et al. (1999) Intake of fatty acids in western Europe with emphasis on trans fatty acids: the TRANSFAIR study. Eur J Clin Nutr 53, $143-157$.

20. World Health Organization (1985) Energy and Protein Reqirements. Report of a Joint FAO/WHO/UNO Expert Consultation. Technical Report Series no. 724. Geneva: WHO.

21. Strand BH \& Tverdal A (2006) Trends in educational inequalities in cardiovascular risk factors: a longitudinal study among 48,000 middle-aged Norwegian men and women. Eur I Epidemiol 21, 731-739.

22. Cox DR (1972) Regression models and life-tables. J R Stat Soc Series B Stat Methodol 34, 187-202.

23. Hu FB, Stampfer MJ, Rimm E, et al. (1999) Dietary fat and coronary heart disease: a comparison of approaches for adjusting for total energy intake and modeling repeated dietary measurements. Am J Epidemiol 149, 531-540.

24. Oh K, Hu FB, Manson JE, et al. (2005) Dietary fat intake and risk of coronary heart disease in women: 20 years of follow-up of the Nurses' Health Study. Am J Epidemiol 161, 672-679.

25. Ascherio A, Rimm EB, Giovannucci EL, et al. (1996) Dietary fat and risk of coronary heart disease in men: cohort follow up study in the United States. Br Med J 313, 84-90.

26. Aro A, Kardinaal AFM, Salminen I, et al. (1995) Adipose-tissue isomeric trans-fatty-acids and risk of myocardial-infarction in 9 countries - the Euramic Study. Lancet 345, 273-278.

27. Pedersen JI, Ringstad J, Almendingen K, et al. (2000) Adipose tissue fatty acids and risk of myocardial infarction a case-control study. Eur J Clin Nutr 54, 618-625.
28. Baylin A, Kabagambe EK, Ascherio A, et al. (2003) High 18:2 trans-fatty acids in adipose tissue are associated with increased risk of nonfatal acute myocardial infarction in Costa Rican adults. J Nutr 133, 1186-1191.

29. Clifton PM, Keogh JB \& Noakes M (2004) Trans fatty acids in adipose tissue and the food supply are associated with myocardial infarction. J Nutr 134, 874-879.

30. Muller H, Seljeflot I, Solvoll K, et al. (2001) Partially hydrogenated soybean oil reduces postprandial $t$-PA activity compared with palm oil. Atherosclerosis 155, 467-476.

31. Almendingen K, Seljeflot I, Sandstad B, et al. (1996) Effects of partially hydrogenated fish oil, partially hydrogenated soybean oil, and butter on hemostatic variables in men. Arterioscler Thromb Vasc Biol 16, 375-380.

32. Mozaffarian D, Cao HM, King IB, et al. (2010) Transpalmitoleic acid, metabolic risk factors, and new-onset diabetes in U.S. adults a cohort study. Ann Intern Med 153, 790-799.

33. Lindenstrom E, Boysen G \& Nyboe J (1994) Influence of total cholesterol, high-density-lipoprotein cholesterol, and triglycerides on risk of cerebrovascular-disease - the Copenhagen City Heart-Study. Br Med J 309, 11-15.

34. Ebrahim S, Sung JH, Song YM, et al. (2006) Serum cholesterol, haemorrhagic stroke, ischaemic stroke, and myocardial infarction: Korean National Health System Prospective Cohort Study. Br Med J 333, 22-25.

35. Ledoux M, Juaneda P \& Sebedio JL (2007) Trans fatty acids: definition and occurrence in foods. Eur J Lipid Sci Technol 109, 891-900.

36. Mozaffarian D (2008) Commentary: ruminant trans fatty acids and coronary heart disease - cause for concern. Int $J$ Epidemiol 37, 182-184. 